\title{
Enseignement
}

\section{Au-delà de la physique classique aux niveaux L1 et L2 ?}

Les enseignements de "Physique Moderne ${ }^{(1)}$ interviennent traditionnellement assez tard dans le cursus scientifique français. Rares sont les universités qui proposent ces enseignements avant le niveau L3, et les programmes des classes préparatoires n'y consacrent que peu de temps ${ }^{(2)}$.

Faut-il continuer à réserver ces enseignements à une minorité de spécialistes, ou bien doit-on considérer qu'ils font partie de la formation scientifique de base, et donc les introduire dès les premières années du Supérieur?

Pour ce qui concerne la filière Classes Préparatoires/ Grandes Écoles, en particulier, la réponse à cette question fait débat. C'est pourquoi l'Union des Professeurs de Spéciales (UPS), association à laquelle nous appartenons, et qui rassemble la très grande majorité des enseignants de chimie, mathématiques et physique des classes préparatoires aux grandes écoles, a entamé depuis plus d'un an une réflexion sur le sujet. Parallèlement, il nous a semblé fructueux d'associer nos collègues universitaires à nos débats, en organisant, le 20 novembre 2010, un colloque intitulé : "Au-delà de la Physique Classique en CPGE ? ". Ce colloque, qui s'est tenu à l'École Nationale Supérieure des Télécommunications à Paris, a réuni environ 90 participants.

La réunion a commencé par les interventions d'Arnaud Le Padellec (UPS Toulouse, SFP), Nicolas Treps (UPMC), Jérôme Pérez (ENSTA-ParisTech), Jean-François Roch (ENS Cachan), Marie-Christine Angonin (UPMC, SYRTE) et Michel Le Bellac (UNS). Leurs présentations ont été suivies d'une table ronde à laquelle participaient également Nicolas Billy (physique) et Johann Yebbou (mathématiques), Inspecteurs Généraux de l'Éducation Nationale.

Les diaporamas complets et le compte rendu de la table ronde peuvent être consultés en ligne à l'adresse : http://sites.google.com/site/physiquemoderneups/. Nous donnons ici quelques-unes des conclusions essentielles que nous avons pu dégager de ces échanges.

De nombreux arguments ont été avancés en faveur d'une introduction précoce de la physique moderne :

- Les cours introductifs de relativité et de mécanique quantique rencontrent toujours un grand succès auprès des étudiants (témoignages de Paris VI et de de physique plus attractifs et à moderniser leur image.

1- Le terme «Physique moderne " désigne ici, à l'exemple anglo-saxon, la physique quantique et la relativité restreinte.

2- Essentiellement quelques aspects de physique atomique et moléculaire, dans le cours de chimie. présentations traditionnelles, ne se limitent pas à un l'ENSTA-ParisTech) et contribuent à rendre les cursus

simple saupoudrage culturel. Les étudiants sont capables de résoudre des exercices à la fois quantitatifs et qualitatifs, de niveau varié, de façon comparable aux domaines de physique classique.

- Le terme «Physique Moderne » est trompeur : ce n'est pas la physique du futur mais celle d'aujourd'hui! De nombreuses applications (lasers, nanotechnologies, GPS...) offrent d'ores et déjà d'importants débouchés économiques.

- Au-delà des applications, la mécanique quantique aussi bien que la relativité restreinte sont, notamment dans leurs développements souvent contre-intuitifs, d'excellentes formations à la rigueur scientifique.

- Il est vraisemblable qu'une dose conséquente de Physique Moderne sera introduite dans les futurs programmes de Terminale S (comme en témoignent les projets publiés à l'heure où nous écrivons), et il semble difficile de ne pas prolonger ces enseignements en CPGE ou en L1 - L2.

Mais cette introduction présente également des risques. L'enseignement de physique en classes préparatoires assure aujourd'hui de larges et solides compétences en physique classique, reconnues par tous. Il ne faudrait pas, dans les suppressions inévitables nécessitées par l'introduction de nouveaux sujets, fragiliser le socle " classique " sur lequel s'appuient ensuite les enseignements dans les Grandes Écoles. Une réflexion est en cours sur ce sujet.

Le débat n'est donc pas clos, et plusieurs prolongements au colloque ont été prévus. Par exemple, un groupe de travail pluridisciplinaire (maths, physique, chimie), regroupant des enseignants de CPGE et de l'Université, a été constitué afin d'approfondir les nouvelles approches de l'enseignement introductif de mécanique quantique.

Nous pourrons donc ainsi continuer à confronter - et rapprocher - les points de vue de deux communautés éducatives qui ont trop peu d'occasions de se rencontrer.

Jean-Aristide Cavaillès ${ }^{(a)}$ (ariscavailles@gmail.com) et Rémi Barbet-Massin ${ }^{(b)}$ (barbet-massin.remi@neuf.fr) (a) Vice-Président de I'UPS, Professeur de Sciences Physiques en CPGE, Lycée Chaptal, 45 bd des Batignolles, 75008 Paris (b) Professeur de Physique en CPGE, Lycée Henri IV, 23 rue Clovis, 75005 Paris 